\title{
Top US universities and institutes for life sciences in 2013
}

\section{Brady Huggett}

sing a ranking of US research institutions based on five years (2009_

2013) of total gross licensing revenue reported by the Association of University Technology Managers, Nature Biotechnology obtained detailed life science commercialization information for 2013, which is shown for major universities (Table 1) and biomedical research institutes (Table 2).
Although the number of licenses executed from this group was down slightly year on year $(-2 \%)$, the number of life science startups increased by $43 \%$, with the University of California system contributing 18 more than in 2012. Life sciences continue to drive startup creation (Fig. 1), with Northwestern University topping the license income earners (Fig. 2).

\begin{tabular}{|c|c|c|c|c|c|}
\hline University & $\begin{array}{l}\text { Licenses and/or } \\
\text { options executed }\end{array}$ & $\begin{array}{l}\text { Gross licensing } \\
\text { revenue received }\end{array}$ & Startups & NIH awards ${ }^{b}$ & NIH funding ${ }^{b}$ \\
\hline University of California system & 236 & $\$ 97,218,208$ & 55 & 4,239 & $\$ 1,741,730,393$ \\
\hline University of Washington/Washington Research Foundation & 134 & $\$ 47,428,701$ & 9 & 932 & $\$ 454,274,167$ \\
\hline Columbia University & 55 & $\$ 137,000,000$ & 6 & 860 & $\$ 348,146,222$ \\
\hline University of Minnesota & 48 & $\$ 34,400,000$ & 9 & 608 & $\$ 264,302,067$ \\
\hline New York University & 29 & $\$ 213,137,273$ & 5 & 612 & $\$ 220,178,414$ \\
\hline Wake Forest University & 23 & $\$ 2,206,625$ & 4 & 242 & $\$ 101,760,292$ \\
\hline Northwestern University & 22 & $\$ 256,163,456$ & 3 & 593 & $\$ 233,095,315$ \\
\hline University of Rochester & 8 & $\$ 27,139,128$ & 0 & 382 & $\$ 146,849,347$ \\
\hline University of Massachusetts & 7 & $\$ 32,624,826$ & 1 & 392 & $\$ 158,659,306$ \\
\hline Princeton University & 4 & $\$ 130,000,000$ & 1 & 117 & $\$ 39,609,228$ \\
\hline
\end{tabular}

aMassachusetts Institute of Technology, Stanford University, University of Wisconsin-Madison and the University of Texas system also ranked highly in gross licensing revenue but could not provide information

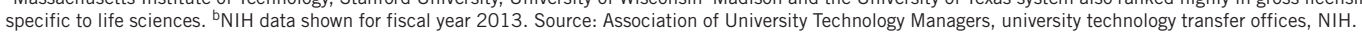

Table 2 US biomedical research institutes ranked by licenses executed, together with revenue, startups and US National Institutes of Health (NIH) awards and funding in 2013.

\begin{tabular}{|c|c|c|c|c|c|}
\hline Research institute & $\begin{array}{l}\text { Licenses and/or } \\
\text { options executed }\end{array}$ & $\begin{array}{l}\text { Gross licensing revenue } \\
\text { received }\end{array}$ & Startups & NIH awards ${ }^{a}$ & NIH funding ${ }^{a}$ \\
\hline Massachusetts General Hospital & 111 & $\$ 75,897,375$ & 14 & 788 & $\$ 339,490,480$ \\
\hline Mayo Foundation for Medical Education and Research & 86 & $\$ 27,778,237$ & 5 & 376 & $\$ 192,248,756$ \\
\hline Brigham \& Women's Hospital & 51 & $\$ 8,015,833$ & 5 & 576 & $\$ 315,919,592$ \\
\hline Memorial Sloan Kettering Cancer Center & 43 & $\$ 148,457,432$ & 2 & 241 & $\$ 111,289,141$ \\
\hline Cleveland Clinic & 38 & $\$ 11,945,033$ & 5 & 203 & $\$ 82,188,005$ \\
\hline Boston Children's Hospital & 35 & $\$ 9,577,933$ & 2 & 303 & $\$ 126,812,298$ \\
\hline Fred Hutchinson Cancer Research Center & 18 & $\$ 10,684,882$ & 0 & 269 & $\$ 199,131,915$ \\
\hline City of Hope and Beckman Research Institute & 11 & $\$ 249,371,883$ & 2 & 85 & $\$ 36,942,940$ \\
\hline Wistar Institute & 6 & $\$ 19,285,000$ & 0 & 55 & $\$ 25,344,586$ \\
\hline Cedars-Sinai Medical Center & 6 & $\$ 12,122,483$ & 1 & 71 & $\$ 27,956,249$ \\
\hline
\end{tabular}

aNIH data shown for fiscal year 2013. Source: Association of University Technology Managers, university technology transfer offices, NIH.

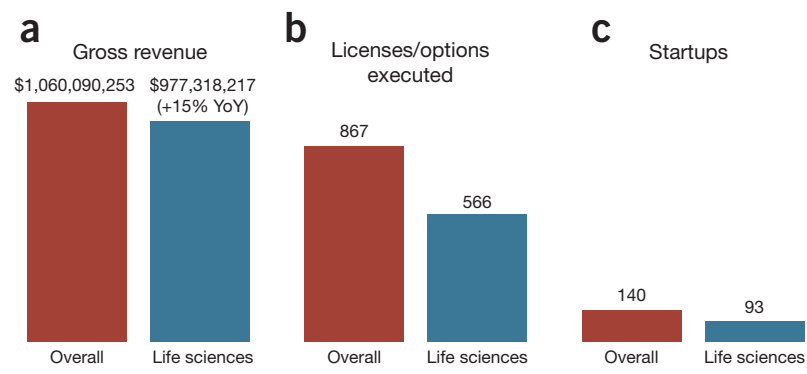

Figure 1 The life sciences accounted for the majority of gross revenue, licenses executed and startups in 2013 (a-c) calculated from our list of selected universities (Table 1). Source: Association of University Technology Managers, university tech transfer offices.

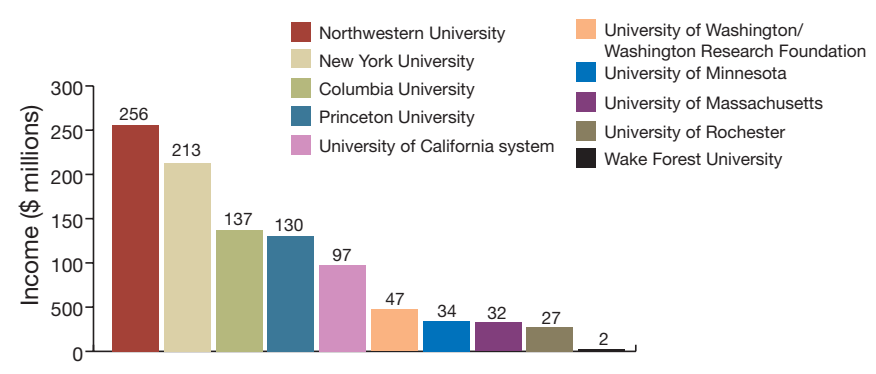

Figure 2 Life science licensing income earners. Source: Association of University Technology Managers, university tech transfer offices.

Brady Huggett is business editor at Nature Biotechnology. 